\section{Utilização e fontes de obtenção de medicamentos: um estudo de base populacional no Município de Campinas, São Paulo, Brasil}

\author{
Use and sources of medicines: a population-based \\ study in Campinas, São Paulo State, Brazil \\ Utilización y fuentes de obtención de medicamentos: \\ un estudio de base poblacional en el municipio de \\ Campinas, São Paulo, Brasil
}

Karen Sarmento Costa 1,2 Priscila Maria Stolses Bergamo Francisco 1 Marilisa Berti de Azevedo Barros 1

\title{
Resumo
}

O uso de medicamentos é influenciado por diferentes fatores. O objetivo foi analisar esse uso e identificar as fontes de obtenção e fatores associados. Estudo transversal de base populacional, amostra por conglomerados, em dois estágios, em Campinas, São Paulo, Brasil, 2008. A prevalência de uso de ao menos um medicamento nos três dias prévios foi de 57,2\%, a de obtenção no Sistema Único de Saúde (SUS) foi de 30\%. Cerca de 47,8\% dos indivíduos que não tinham plano precisaram desembolsar para adquirir os medicamentos e 10,9\% dos que tinham obtiveram no SUS. Utilizaram com maior frequência os medicamentos do SUS os indivíduos com mais idade, viúvos, pretos/pardos, com menor escolaridade e renda, $e$ que não tinham plano. Apenas 2,1\% da população entrevistada referiram não ter conseguido obter o medicamento que lhe havia sido prescrito, $e$ os principais motivos foram não ter no sistema público e a falta de recurso. O estudo identifica os segmentos com maior obtenção de medicamentos do SUS e revela que estes subgrupos apresentam porcentuais baixos de uso no sistema.

Assistência Farmacêutica; Uso de Medicamentos; Inquéritos

Epidemiológicos

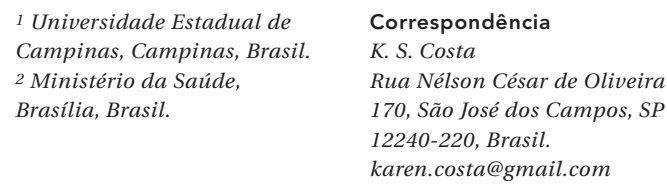

1 Universidade Estadual de Campinas, Campinas, Brasil.

2 Ministério da Saúde, K. S. Costa

Rua Nélson César de Oliveira 170, São José dos Campos, SP 12240-220, Brasil.

karen.costa@gmail.com 


\section{Introdução}

O acesso aos medicamentos envolve uma complexa rede de atores públicos e privados e várias combinações de provisão de serviços e de financiamentos estatal e privado. Os agentes e agências envolvidos no acesso ao medicamento desempenham diferentes papéis, relacionados ao contexto econômico, político e social e aos modelos e sistemas de saúde dos diferentes países nos quais são fomentadas as condições para que tal acesso se efetive 1 .

Não existe na literatura um consenso sobre uma definição operacional de acesso a medicamentos, e tampouco sobre um modelo de avaliação conclusivo que permitisse a comparação do desempenho, neste tema, entre os diferentes países 2. Segundo a Organização Mundial da Saúde (OMS), o acesso aos medicamentos é viabilizado mediante a disponibilidade dos fármacos, a capacidade aquisitiva das pessoas, a acessibilidade geográfica, a sua aceitabilidade e as condições de uso racional, sendo este acesso reconhecido pela Organização das Nações Unidas (ONU) como um dos cinco indicadores selecionados para avaliar os avanços na garantia do direito à saúde 3 .

Apesar do advento de políticas públicas voltadas a favorecer o acesso em diferentes países, como também da generalização de informações nas sociedades a respeito do tema, o acesso a medicamentos permanece problemático, em escala mundial, para populações pobres e vulneráveis 4 . Nos países periféricos, estima-se que a disponibilidade média de medicamentos seja de $35 \%$ em unidades públicas e $66 \%$ no setor privado. Em países que apresentam um numeroso contingente populacional com renda baixa e média, verifica-se também a inexistência de sistemas informatizados com capacidade de integrar os dados de saúde relacionados aos medicamentos 5 .

O Estado brasileiro incorporou, dentre seus princípios e diretrizes constitucionais, a garantia da assistência terapêutica integral, inclusive a farmacêutica a ser concretizada por meio da implantação de políticas, programas e ações relativos ao campo farmacêutico, mediante pactuação com os diferentes gestores do Sistema Único de Saúde (SUS) em âmbito interfederativo. Dentre as políticas nacionais, mencionam-se os seguintes instrumentos do arcabouço institucional que refletem a organização do Estado, em busca de prover o acesso ao medicamento com uso racional: a Política Nacional de Medicamentos (PNM) 6, de 1998; a Lei de Genéricos 7, de 1999; a Política Nacional de Assistência Farmacêutica (PNAF) 8, de 2004; o Programa Farmácia Popular 9, de 2004; e mais recentemente, o Pro- grama Nacional de Qualificação da Assistência Farmacêutica no SUS 10.

Estudos brasileiros têm evidenciado, em geral, o maior uso de medicamentos pelas mulheres 11,12,13,14, pelos indivíduos mais idosos 11,12,14 e com maior número de doenças crônicas 12,13,15. A literatura evidencia também diferenças de consumo segundo o nível socioeconômico 11,13 e segundo raça/cor da pele 11,14. As pesquisas epidemiológicas brasileiras que abordam o acesso e uso de medicamentos ainda são escassas e utilizam diferentes métodos e populações alvo, o que dificulta a comparação de seus resultados e a avaliação de impactos dos novos direcionamentos dados para as políticas farmacêuticas do país 13,14.

Considerando que os inquéritos populacionais são instrumentos valiosos para a obtenção de informações relativas ao acesso e uso de medicamentos 15 , e a necessidade de conhecer melhor o padrão de utilização e as fontes de obtenção dos medicamentos pelos diferentes segmentos sociais da população, o presente trabalho teve o propósito de analisar a prevalência do uso de medicamentos, segundo características sociodemográficas, de condições de saúde e descritoras do uso de serviços de saúde na população adulta (20 anos ou mais) do Município de Campinas, São Paulo, Brasil, identificar a fonte de obtenção dos medicamentos, analisar os fatores associados à obtenção de medicamentos no SUS e identificar para os medicamentos não obtidos a quais grupos pertencem e os principais motivos relatados.

\section{Métodos}

\section{Área e população de estudo}

Estudo transversal de base populacional que utilizou os dados do Inquérito de Saúde de Campinas (ISACamp 2008/2009), realizado pelo Centro Colaborador em Análise de Situação de Saúde (CCAS) do Departamento de Saúde Coletiva, Faculdade de Ciências Médicas, Universidade Estadual de Campinas (FCM/UNICAMP).

A pesquisa visou à obtenção de informações sobre três domínios de idade: adolescentes (10 a 19 anos), adultos (20 a 59) e idosos (60 anos ou mais), residentes em área urbana do Município de Campinas. A amostra foi obtida por meio de amostragem probabilística, por conglomerados e em dois estágios, considerando os setores censitários como unidade primária. No primeiro estágio, 50 setores censitários foram sorteados com probabilidade proporcional ao tamanho, dado pelo número de domicílios do setor. No segun- 
do, os domicílios foram selecionados por sorteio sistemático aplicado às relações de domicílios que haviam sido elaboradas para cada um dos setores sorteados.

O número de pessoas para compor a amostra foi obtido considerando-se a situação correspondente à máxima variabilidade para a frequência dos eventos estudados $(p=0,50)$, coeficiente de confiança de $95 \%$ na determinação dos intervalos de confiança $(z=1,96)$, erro de amostragem entre 4 e 5 pontos percentuais e efeito de delineamento igual a 2, totalizando mil indivíduos em cada domínio de idade. Esperando-se uma taxa de $80 \%$ de resposta, o tamanho da amostra foi corrigido para 1.250 e, para obter este número de amostra em cada domínio, após atualização em campo dos mapas dos setores sorteados e elaboração da lista de endereços, foram selecionados de forma independente 2.150, 700 e 3.900 domicílios para adolescentes, adultos e idosos, respectivamente.

A descrição do plano de amostragem do inquérito está disponível em Alves 16. Neste estudo foram analisados dados relativos à população adulta de 20 anos e mais $(\mathrm{n}=2.476)$.

\section{Variáveis e coletas de dados}

A coleta de dados ocorreu nos domicílios, com entrevistas face a face realizadas por entrevistadores treinados e supervisionados, com base em questionário estruturado, previamente testado em estudo piloto. Em cada residência foram entrevistados todos os moradores da faixa etária selecionada para aquele domicílio.

As variáveis dependentes analisadas no presente trabalho foram: o uso de ao menos um medicamento nos três dias que antecederam a entrevista e a fonte de obtenção dos medicamentos utilizados baseando-se em duas categorizações: a primeira considerando as categorias farmácias do SUS, drogarias e farmácias do setor privado (D\&F/Outros) e forma mista para identificar a relevância com que cada uma destas alternativas se apresenta, na realidade pesquisada; e a segunda, farmácias do SUS e demais (D\&F/Outros e mistas) para avaliar os fatores associados à obtenção exclusiva de medicamentos pelo SUS.

A identificação dos indivíduos que referiram uso de ao menos um medicamento nos três dias foi investigada pelas perguntas: (1) "Você utilizou algum medicamento nos últimos três dias?”; (2) "Quantos medicamentos?", “Quais?”.

A identificação das fontes de obtenção dos medicamentos, dos indivíduos que referiram uso de ao menos um medicamento nos três dias que antecederam a pesquisa $(n=1.732)$ foi investigada por meio das seguintes perguntas feitas em relação a cada um dos medicamentos consumidos: "Você pagou pelo remédio?". As possibilidades de resposta eram: não; sim, parcialmente; sim, integralmente; não sei/não quis responder (NS/NR). Com exceção dos que haviam respondido "sim, integralmente", os demais respondiam a uma segunda pergunta: “Quem cobriu os gastos?", cujas respostas possíveis eram: SUS - centro de saúde; SUS - outro local; Programa Farmácia Popular; plano individual de saúde; já tinha o medicamento; outro; NS/NR.

Com base nessas duas perguntas foi elaborada a variável fonte de obtenção dos medicamentos utilizados, com as categorias: medicamentos obtidos no SUS (não pagou por nenhum dos medicamentos que utilizou e todos foram fornecidos pelo SUS); obteve em farmácias e drogarias (pagou integralmente por todos os medicamentos que utilizou); formas mistas: os medicamentos que usou foram obtidos em fontes diferentes; e demais: pagaram parcialmente pelo(s) medicamento(s), já tinham ou obtiveram de vizinhos, amigos ou instituições.

A variável fonte de obtenção possibilitou elaborar a variável obteve do SUS todos os medicamentos que utilizou com alternativas: sim e não.

Como variáveis independentes foram consideradas:

a) Demográficas e socioeconômicas: sexo, faixa etária, situação conjugal, cor da pele/raça autorreferida, escolaridade (em anos de estudos), renda familiar per capita (em salários mínimos) e filiação a plano médico de saúde.

b) Indicadores de condições de saúde e de uso de serviços de saúde: número de doenças crônicas (considerando-se hipertensão arterial, diabetes, doença cardiovascular, tumor/câncer, reumatismo/artrite/artrose, osteoporose, asma/bronquite/enfisema, tendinite/LER/DORT, problemas circulatórios e outros), número de problemas de saúde (considerando-se dor de cabeça/enxaqueca, dor nas costas/problemas de coluna, alergia, problema emocional, tontura/vertigem, insônia, problema urinário), procura por serviço ou profissional de saúde para problemas relacionados à saúde nas duas semanas anteriores à pesquisa e número de medicamentos utilizados nos três dias prévios à entrevista.

$\mathrm{Na}$ avaliação do não acesso, levando-se em conta todos os adultos com indicação médica para uso de algum medicamento nos 15 dias que antecederam a pesquisa, foram estimadas as frequências relativas para os principais motivos relatados e identificados os tipos de medicamentos. Dentre esses motivos, as respostas possíveis eram: não tinha no sistema público; não tinha 
recurso; o plano não cobria; não encontrei nas farmácias; outro, especifique; NS/NR.

\section{Análise de dados}

Foram estimadas as prevalências do uso de medicamentos nos últimos três dias e de obtenção de medicamentos, segundo fontes de obtenção. Para verificar a associação entre as diversas variáveis e a fonte de obtenção de medicamentos foram utilizados o teste qui-quadrado de Pearson e o teste de tendência linear, ambos com nível de significância de $5 \%$. As variáveis que apresentaram valor de $\mathrm{p}<0,20$ para o teste de associação foram incluídas na análise multivariada, realizada pela regressão de Poisson 17 obedecendo ao seguinte modelo hierárquico: na primeira etapa foram incluídas as características sociodemográficas associadas ao acesso total aos medicamentos utilizados no SUS e, na segunda, além das variáveis pertencentes ao primeiro bloco que mantiveram significância após ajuste pelas demais variáveis do mesmo nível hierárquico, foram acrescentadas as que se referiam às condições de saúde e uso de serviços de saúde. No modelo final restaram aquelas que apresentaram um valor de $\mathrm{p}<0,05$, quando ajustadas pelas variáveis do nível superior e do mesmo nível. Todas as análises foram realizadas considerando-se o efeito do desenho amostral para análises de inquéritos baseados em delineamentos complexos 18 do programa Stata 11.0 (StataCorp LP, College Station, Estados Unidos), que consideram os fatores de ponderação.

\section{Procedimentos éticos}

Os indivíduos sorteados foram informados sobre os objetivos da pesquisa e aqueles que concordaram em participar assinaram o Termo de Consentimento Livre e Esclarecido. O projeto de pesquisa foi aprovado pelo Comitê de Ética da UNICAMP, em adendo ao parecer no 079/2007.

\section{Resultados}

A prevalência de uso de ao menos um medicamento, nos três dias que antecederam a pesquisa na população acima de 20 anos residente em Campinas, foi de 57,2\% (IC95\%: 53,7-60,7).

A distribuição da população segundo indicadores sociodemográficos e de saúde, assim como as prevalências de uso de medicamentos, estão apresentadas na Tabela 1. A população estudada é composta predominantemente por mulheres, indivíduos entre 30 e 59 anos, casados/unidos e que referiram cor da pele branca e com mais de 9 anos de escolaridade.

A prevalência do uso de medicamentos foi maior nas mulheres, e com o aumento da idade revelou-se crescente conforme o aumento do número de doenças crônicas, sendo superior nos indivíduos que procuraram serviços de saúde nas duas últimas semanas. Entre os menos escolarizados e com menor renda, assim como entre os indivíduos que referiram ter plano de saúde privado, as prevalências de uso de medicamentos também foram superiores (Tabela 1). Observou-se para todas as doenças/condições crônicas e problemas de saúde prevalência de uso de medicamentos superior a $63 \%$, sendo que nos indivíduos que referiram diabetes, hipertensão arterial, reumatismo/artrite/artrose e osteoporose o uso maior do que $90 \%$.

Na população estudada, segundo demonstrado na Tabela 2, foi constatado que, entre os indivíduos que haviam consumido medicamentos nos três dias prévios à entrevista, 30\% obtiveram todos os medicamentos que utilizaram em serviços do SUS $(n=625)$, cerca $63 \%$ em drogarias e farmácias ( $n=941)$ e $7 \%(n=126)$ conseguiram em diferentes fontes entre os medicamentos de que fizeram uso. A fonte de obtenção do medicamento revelou-se associada com as variáveis: faixa etária, situação conjugal, cor da pele, renda, escolaridade e posse de plano de saúde. Entre os pacientes que tinham plano privado de saúde, $10,9 \%$ obtiveram no SUS os medicamentos que necessitavam.

Entre as variáveis relativas às condições de saúde e uso de serviços de saúde observa-se uma elevação da proporção de indivíduos que acessam o SUS para obtenção de medicamentos com o aumento do número de doenças crônicas e de medicamentos utilizados $(\mathrm{p}<0,05)$ (Tabela 2). Na avaliação das doenças/condições crônicas referidas específicas, a obtenção dos medicamentos no SUS foi significativamente maior entre hipertensos, diabéticos e com problemas circulatórios, comparativamente aos que não tinham estes problemas. Para os problemas de saúde investigados, maior prevalência de obtenção no SUS foi verificada para os que relataram dor de cabeça frequente/enxaqueca e questões emocionais.

A Tabela 2 apresenta a razão de prevalência de obtenção de medicamentos no SUS, considerando os indivíduos que conseguiram nestes serviços todos os medicamentos que utilizaram nos três dias que antecederam a entrevista. Verificase nessa tabela o aumento da prevalência com a idade, maiores prevalências entre os viúvos, nos que referiram cor da pele não branca e entre os que não tinham plano de saúde, e gradiente 
Tabela 1

Distribuição da amostra e prevalência do uso de medicamentos, segundo variáveis sociodemográficas e de saúde. Campinas, São Paulo, Brasil, 2008.

\begin{tabular}{|c|c|c|c|c|c|}
\hline \multirow[t]{2}{*}{ Variáveis } & \multicolumn{2}{|c|}{ Amostra } & \multicolumn{2}{|c|}{ Prevalência de uso } & \multirow[t]{2}{*}{ Valor de $p$ * } \\
\hline & $\mathrm{n}$ & $\%$ & $\mathrm{n}$ & $\%$ & \\
\hline Sexo & & & & & $<0,001$ \\
\hline Masculino & 1.064 & 47,71 & 627 & 45,44 & \\
\hline Feminino & 1.412 & 52,29 & 1.105 & 68,04 & \\
\hline Idade (anos) & & & & & $<0,001$ \\
\hline $20-29$ & 305 & 27,23 & 137 & 44,18 & \\
\hline $30-59$ & 652 & 56,67 & 373 & 56,98 & \\
\hline $60-69$ & 820 & 8,69 & 622 & 75,53 & \\
\hline 70 ou mais & 699 & 7,41 & 600 & 86,13 & \\
\hline Situação conjugal & & & & & $<0,001$ \\
\hline Casado/Unido & 1.441 & 60,97 & 997 & 59,17 & \\
\hline Solteiro/Separado & 568 & 33,08 & 337 & 49,30 & \\
\hline Viúvo & 467 & 5,95 & 398 & 81,88 & \\
\hline Cor & & & & & 0,158 \\
\hline Branca & 1.854 & 73,88 & 1.324 & 58,60 & \\
\hline Não branca & 618 & 26,12 & 406 & 53,51 & \\
\hline Escolaridade (anos) & & & & & $<0,01$ \\
\hline $0-4$ & 1.135 & 22,70 & 905 & 71,52 & \\
\hline $5-8$ & 408 & 21,97 & 251 & 54,87 & \\
\hline $9-11$ & 436 & 26,79 & 268 & 52,99 & \\
\hline 12 e mais & 496 & 28,54 & 307 & 51,76 & \\
\hline Renda mensal per capita (salários mínimos) & & & & & 0,300 \\
\hline$\leq 1$ & 1.020 & 40,54 & 708 & 54,97 & \\
\hline$>1 \mathrm{a} \leq 3$ & 997 & 39,72 & 700 & 59,83 & \\
\hline$>3$ & 459 & 19,74 & 324 & 56,76 & \\
\hline Plano de saúde & & & & & 0,084 \\
\hline Não possui & 1.347 & 55,31 & 892 & 54,84 & \\
\hline Possui & 1.126 & 44,69 & 839 & 60,24 & \\
\hline Número de doenças crônicas & & & & & $<0,001$ \\
\hline 0 & 883 & 56,26 & 332 & 38,85 & \\
\hline 1 & 582 & 24,04 & 445 & 69,67 & \\
\hline 2 ou mais & 979 & 19,70 & 925 & 92,40 & \\
\hline \multicolumn{6}{|l|}{ Relato das seguintes doenças/condições crônicas } \\
\hline Hipertensão arterial & 957 & 20,40 & 924 & 92,80 & $<0,001$ \\
\hline Diabetes & 370 & 6,66 & 358 & 97,04 & $<0,001$ \\
\hline Doença cardiovascular & 303 & 5,81 & 286 & 84,80 & $<0,001$ \\
\hline Reumatismo/Artrite/Artrose & 435 & 7,15 & 402 & 92,97 & $<0,001$ \\
\hline Osteoporose & 241 & 3,18 & 226 & 92,57 & $<0,001$ \\
\hline Asma/Bronquite/Enfisema & 126 & 3,85 & 107 & 76,17 & $<0,001$ \\
\hline Problemas circulatórios & 492 & 11,72 & 439 & 82,52 & $<0,001$ \\
\hline \multicolumn{6}{|l|}{ Problemas de saúde (queixas) } \\
\hline Dor de cabeça frequente/Enxaqueca & 563 & 26,35 & 436 & 69,74 & $<0,001$ \\
\hline Dor nas costas/Problemas de coluna & 970 & 33,18 & 783 & 70,30 & $<0,001$ \\
\hline Alergia & 587 & 26,75 & 436 & 63,04 & 0,008 \\
\hline Problema emocional & 527 & 19,32 & 467 & 81,27 & $<0,001$ \\
\hline Tontura/Vertigem & 383 & 10,66 & 336 & 82,72 & $<0,001$ \\
\hline Insônia & 494 & 16,13 & 428 & 80,60 & $<0,001$ \\
\hline Problema urinário & 190 & 4,17 & 168 & 84,87 & 0,003 \\
\hline Procura por serviço de saúde nas últimas duas & & & & & $<0,001$ \\
\hline \multicolumn{6}{|l|}{ semanas } \\
\hline Não & 1.920 & 80,43 & 1.239 & 51,52 & \\
\hline Sim & 556 & 19,57 & 492 & 81,09 & \\
\hline
\end{tabular}

n: número de indivíduos na amostra não ponderada.

* Valor de $p$ do teste qui-quadrado. 
Tabela 2

Prevalência e razão de prevalência (RP) do acesso aos medicamentos em diferentes fontes de obtenção, segundo variáveis sociodemográficas e de saúde na população adulta. Campinas, São Paulo, Brasil, 2008.

\begin{tabular}{|c|c|c|c|c|c|c|}
\hline \multirow[t]{2}{*}{ Variável } & \multicolumn{2}{|c|}{ SUS } & \multicolumn{2}{|c|}{ Não SUS } & \multirow[t]{2}{*}{ RP bruta } & \multirow[t]{2}{*}{ Valor de $p$ * } \\
\hline & $\mathbf{n}$ & $\%$ & $\mathbf{n}$ & $\%$ & & \\
\hline \multicolumn{7}{|l|}{ Sexo } \\
\hline Masculino & 234 & 28,00 & 393 & 72,00 & 1,00 & 0,271 \\
\hline Feminino & 391 & 31,21 & 714 & 68,79 & $1,11(0,92-1,35)$ & \\
\hline \multicolumn{7}{|l|}{ Idade (anos) ** } \\
\hline $20-29$ & 27 & 19,06 & 110 & 80,94 & 1,00 & \\
\hline $30-59$ & 119 & 30,76 & 254 & 69,24 & $1,61(1,13-2,31)$ & 0,010 \\
\hline $60-69$ & 243 & 38,10 & 379 & 61,90 & $2,00(1,40-2,85)$ & $<0,001$ \\
\hline 70 ou mais & 236 & 38,50 & 364 & 61,50 & $2,02(1,35-3,02)$ & 0,001 \\
\hline \multicolumn{7}{|l|}{ Situação conjugal } \\
\hline Casado/Unido & 339 & 28,43 & 658 & 71,57 & 1,00 & \\
\hline Separado/Solteiro & 122 & 29,92 & 215 & 70,08 & $0,95(0,75-1,21)$ & 0,674 \\
\hline Viúvo & 164 & 41,91 & 234 & 58,09 & $1,40(1,10-1,78)$ & 0,007 \\
\hline \multicolumn{7}{|l|}{ Cor } \\
\hline Branca & 410 & 26,57 & 914 & 73,43 & 1,00 & \\
\hline Não branca & 215 & 40,84 & 191 & 59,16 & $1,54(1,19-1,98)$ & 0,001 \\
\hline \multicolumn{7}{|l|}{ Escolaridade (anos) } \\
\hline $0-4$ & 448 & 52,60 & 457 & 47,40 & $6,26(3,98-9,82)$ & \\
\hline $5-8$ & 97 & 36,70 & 154 & 63,30 & $4,36(2,50-7,61)$ & $<0,01$ \\
\hline $9-11$ & 51 & 21,00 & 217 & 79,00 & $2,50(1,56-4,00)$ & $<0,01$ \\
\hline 12 e mais & 29 & 8,41 & 278 & 91,59 & 1,00 & $<0,01$ \\
\hline \multicolumn{7}{|l|}{ Renda mensal per capita (salários } \\
\hline \multicolumn{7}{|l|}{ minimos) $* \star$} \\
\hline$\leq 1$ & 331 & 40,16 & 377 & 59,84 & $4,54(2,90-7,11)$ & \\
\hline$>1 \mathrm{a} \leq 3$ & 259 & 30,44 & 441 & 69,56 & $3,44(2,17-5,46)$ & $<0,01$ \\
\hline$>3$ & 35 & 8,85 & 289 & 91,15 & 1,00 & $<0,01$ \\
\hline \multicolumn{7}{|l|}{ Plano de saúde } \\
\hline Possui & 123 & 10,91 & 716 & 89,09 & 1,00 & \\
\hline Não possui & 502 & 46,93 & 390 & 53,07 & $4,30(3,17-5,84)$ & $<0,001$ \\
\hline \multicolumn{7}{|l|}{ Número de doenças crônicas } \\
\hline 0 & 61 & 18,35 & 271 & 81,65 & 1,00 & \\
\hline 1 & 164 & 31,26 & 281 & 68,74 & $1,70(1,24-2,33)$ & 0,001 \\
\hline 2 ou mais & 387 & 41,36 & 538 & 58,64 & $2,25(1,72-2,96)$ & $<0,001$ \\
\hline \multicolumn{7}{|c|}{ Relato das seguintes doenças/condições } \\
\hline \multicolumn{7}{|l|}{ crônicas } \\
\hline Hipertensão arterial & 435 & 46,22 & 489 & 53,78 & $2,10(1,70-2,60)$ & $<0,001$ \\
\hline Diabetes & 191 & 56,70 & 167 & 43,31 & $2,16(1,78-2,61)$ & $<0,001$ \\
\hline Doença cardiovascular & 92 & 34,89 & 194 & 65,11 & $1,18(0,89-1,56)$ & 0,233 \\
\hline Reumatismo/Artrite/Artrose & 130 & 33,08 & 272 & 66,92 & $1,11(0,81-1,53)$ & 0,488 \\
\hline Osteoporose & 70 & 31,76 & 156 & 68,25 & $1,06(0,78-1,44)$ & 0,686 \\
\hline Asma/Bronquite/Enfisema & 37 & 32,44 & 70 & 67,56 & $1,08(0,74-1,60)$ & 0,676 \\
\hline Problemas circulatórios & 179 & 43,53 & 260 & 56,47 & $1,60(1,29-1,98)$ & $<0,001$ \\
\hline
\end{tabular}

(continua) 
Tabela 2 (continuação)

\begin{tabular}{|c|c|c|c|c|c|c|}
\hline \multirow[t]{2}{*}{ Variável } & \multicolumn{2}{|c|}{ SUS } & \multicolumn{2}{|c|}{ Não SUS } & \multirow[t]{2}{*}{ RP bruta } & \multirow[t]{2}{*}{ Valor de $p$ * } \\
\hline & $\mathbf{n}$ & $\%$ & $\mathbf{n}$ & $\%$ & & \\
\hline \multicolumn{7}{|l|}{ Problemas de saúde (queixas) } \\
\hline Dor de cabeça frequente/Enxaqueca & 191 & 34,65 & 245 & 65,35 & $1,25(1,01-1,55)$ & 0,045 \\
\hline Dor nas costas/Problemas de coluna & 292 & 33,01 & 491 & 66,99 & $1,18(0,92-1,51)$ & 0,181 \\
\hline Alergia & 137 & 28,01 & 299 & 71,99 & $0,91(0,73-1,13)$ & 0,381 \\
\hline Problema emocional & 153 & 37,25 & 314 & 62,75 & $1,37(1,11-1,68)$ & 0,004 \\
\hline Tontura/Vertigem & 130 & 34,78 & 206 & 65,22 & $1,19(0,90-1,59)$ & 0,219 \\
\hline Insônia & 155 & 33,20 & 273 & 66,80 & $1,14(0,92-1,41)$ & 0,211 \\
\hline Problema urinário & 52 & 30,12 & 116 & 69,88 & $1,00(0,61-1,65)$ & 0,986 \\
\hline Procura por serviço de saúde nas últimas & & & & & & 0,411 \\
\hline \multicolumn{7}{|l|}{ duas semanas } \\
\hline Não & 439 & 29,09 & 810 & 70,91 & 1,00 & \\
\hline Sim & 186 & 32,43 & 297 & 67,57 & $1,11(0,86-1,45)$ & \\
\hline \multicolumn{7}{|l|}{ Número de medicamentos utilizados ** } \\
\hline 1 & 141 & 20,65 & 424 & 79,35 & 1,00 & \\
\hline 2 & 164 & 31,69 & 236 & 68,31 & $1,53(1,18-1,99)$ & 0,002 \\
\hline 3 & 130 & 47,57 & 127 & 52,43 & $2,30(1,69-3,14)$ & $<0,001$ \\
\hline 4 ou mais & 190 & 43,75 & 320 & 56,25 & $2,12(1,60-2,81)$ & $<0,001$ \\
\hline
\end{tabular}

SUS: Sistema Único de Saúde.

* Teste de Wald;

** Valor de $p<0,01$ para o teste de tendência linear.

positivo de aumento da prevalência com a redução da escolaridade e da renda. Também foi observada maior prevalência com o aumento do número de doenças crônicas entre os hipertensos e diabéticos e nos que referiram o uso de 2 ou mais medicamentos.

No modelo de regressão múltipla hierárquica, as variáveis sociodemográficas, faixa etária, escolaridade, renda familiar per capita e plano de saúde mantiveram-se estatisticamente associadas à obtenção de medicamentos do SUS. No bloco relativo às condições de saúde, número de doenças crônicas e de medicamentos utilizados estiveram positivamente associados à obtenção de medicamentos pelo SUS quando ajustados entre si e para as variáveis que persistiram no bloco anterior (Tabela 3).

$\mathrm{Na}$ população pesquisada em Campinas, apenas 2,1\% (IC95\%: 1,3-3,0) referiram não ter conseguido algum medicamento prescrito por médico nos 15 dias anteriores à pesquisa. Os principais motivos relatados para a falta de acesso foram: "não tinha no sistema público" $(42,5 \%)$ e "não tinha recurso" (35,9\%). Dentre os tipos de medicamentos não acessados foram observadas maiores frequências nos grupos de medicamentos para o sistema cardiovascular (15) e do trato alimentar (3).

\section{Discussão}

Os principais resultados deste trabalho apontaram que menos da metade dos indivíduos obteve seus medicamentos exclusivamente junto ao SUS, sendo estes de menor renda, escolaridade e não cobertos por plano de saúde, enfatizando a importância da assistência farmacêutica na redução da desigualdade no acesso ao medicamento. Indivíduos em piores condições de saúde obtiveram também mais frequentemente seus medicamentos junto ao SUS, o que sugere o papel da assistência farmacêutica pública na redução da iniquidade.

A prevalência de uso de medicamentos pela população de 20 anos ou mais, nos três dias prévios à realização da pesquisa no Município de Campinas, foi de 57,2\%. Outro estudo, realizado em Campinas em 2001/2002, havia encontrado uma prevalência de 48,5\% na população com 18 anos ou mais 14. A etapa brasileira da Pesquisa Mundial de Saúde (PMS) 19, realizada em 2003, na qual observou-se na população acima de 18 anos que referiram uso de algum medicamento nas duas semanas prévias à entrevista, constatou-se que quase a metade desta população referiu o uso (49\%).

No entanto, outros trabalhos apresentaram prevalências de acesso superiores às encontra- 
Tabela 3

Modelo de regressão múltipla do acesso aos medicamentos no Sistema Único de Saúde (SUS), segundo variáveis sociodemográficas e de saúde. Campinas, São Paulo, Brasil, 2008.

\begin{tabular}{|c|c|c|c|c|c|c|}
\hline \multirow[t]{2}{*}{ Variável } & \multicolumn{3}{|c|}{ Primeira etapa * } & \multicolumn{3}{|c|}{ Segunda etapa ** } \\
\hline & RP & IC95\% & Valor de p & $\mathrm{RP}$ & IC95\% & Valor de $p$ \\
\hline \multicolumn{7}{|l|}{ Idade (anos) } \\
\hline $20-29$ & 1,00 & & & 1,00 & & \\
\hline $30-59$ & 1,36 & $1,00-1,87$ & 0,051 & 1,11 & $0,76-1,61$ & 0,580 \\
\hline $60-69$ & 1,42 & $1,05-1,95$ & 0,025 & 0,95 & $0,64-1,41$ & 0,802 \\
\hline 70 ou mais & 1,44 & $1,02-2,05$ & 0,040 & 0,92 & $0,60-1,42$ & 0,707 \\
\hline \multicolumn{7}{|c|}{ Escolaridade (anos) } \\
\hline 12 e mais & 1,00 & & & 1,00 & & \\
\hline $9-11$ & 1,71 & $1,03-2,84$ & 0,037 & 1,64 & $1,01-2,65$ & 0,046 \\
\hline $5-8$ & 2,24 & $1,24-4,07$ & 0,009 & 2,12 & $1,21-3,70$ & 0,009 \\
\hline $0-4$ & 3,01 & $1,84-4,93$ & $<0,001$ & 2,65 & $1,61-4,36$ & $<0,001$ \\
\hline \multicolumn{7}{|c|}{ Renda mensal per capita (salários mínimos) } \\
\hline$>3$ & 1,00 & & & 1,00 & & \\
\hline$>1 a \leq 3$ & 1,47 & $0,96-2,24$ & 0,073 & 1,63 & $1,02-2,59$ & 0,041 \\
\hline$\leq 1$ & 1,55 & $1,06-2,28$ & 0,024 & 1,67 & $1,08-2,59$ & 0,022 \\
\hline \multicolumn{7}{|l|}{ Plano de saúde } \\
\hline Possui & 1,00 & & & 1,00 & & \\
\hline Não possui & 2,95 & $2,18-3,98$ & $<0,001$ & 2,95 & $2,16-4,02$ & 0,001 \\
\hline \multicolumn{7}{|c|}{ Número de doenças crônicas } \\
\hline 0 & & & & 1,00 & & \\
\hline 1 & & & & 1,39 & $1,02-1,91$ & 0,040 \\
\hline 2 ou mais & & & & 1,49 & $1,08-2,05$ & 0,016 \\
\hline \multicolumn{7}{|c|}{ Número de medicamentos utilizados } \\
\hline 1 & & & & 1,00 & & \\
\hline 2 & & & & 1,43 & $1,15-1,78$ & 0,002 \\
\hline 3 & & & & 1,73 & $1,33-2,24$ & $<0,001$ \\
\hline 4 ou mais & & & & 1,77 & $1,32-2,37$ & $<0,001$ \\
\hline
\end{tabular}

IC95\%: intervalo de 95\% de confiança; RP: razão de prevalência.

* RP ajustadas pelas variáveis sociodemográficas;

** RP ajustadas por todas as variáveis da tabela.

das no Município de Campinas, a exemplo de estudo realizado na cidade de Florianópolis, Santa Catarina, Brasil, no qual foi verificada uma prevalência de acesso a medicamentos, por parte da população acima de 20 anos de idade em trinta dias prévios à entrevista, de 76,5\% 20. Em estudos internacionais têm sido observadas prevalências de uso de medicamentos que variam entre $36,8 \%$ e $67,4 \% 21$. A variabilidade de resultados pode decorrer do segmento etário estudado, do tipo de medicamento investigado, de diferenças metodológicas, dos períodos recordatórios e das políticas farmacêuticas vigentes 22 .

Os fatores significativamente associados ao maior uso de medicamentos, verificados no presenteestudo, apresentaramsimilaridadescomou- tros trabalhos realizados no país: maior frequência de uso de medicamentos entre mulheres 11,12,13,14, em indivíduos mais idosos 11,12,14,20,21, dentre pessoas com maior número de doenças crônicas 12,15,19,22, entre os que referiram morbidade e que procuraram serviços de saúde nas duas últimas semanas 12,13.

As mulheres demonstram maior preocupação com a saúde e procuram com maior frequência os serviços de saúde, sendo que estes têm programas voltados, prioritariamente, à saúde da mulher. As outras associações observadas são resultados esperados, uma vez que indivíduos acometidos pelo maior número de doenças crônicas, e também com o avanço da idade, procuram mais frequentemente os serviços de saúde, 
condição que tende a exercer influência no uso de medicamentos.

Observou-se que a significativa maior utilização de medicamentos entre indivíduos menos escolarizados, quando comparados aos de maior escolaridade, não é comum na literatura. Em geral, os estudos farmacoepidemiológicos apontam maior prevalência de uso de medicamentos entre pessoas mais escolarizadas 11,23. No entanto, o estudo da Pesquisa Nacional por Amostra de Domicílios (PNAD 2008) 22 apontou maior utilização de medicamentos entre indivíduos com até 4 anos de escolaridade, com uma frequência de $46,7 \%$, inferior à encontrada no presente estudo que foi de $71,5 \%$.

As prevalências de uso de medicamentos apresentaram patamares elevados entre os indivíduos que relataram a presença de doenças crônicas. Considerando que, para o conjunto dessas condições, a utilização de medicamentos é fundamental para o alcance dos resultados terapêuticos, torna-se imprescindível garantir o acesso aos medicamentos e às orientações para o uso adequado, por meio de serviços clínicos integrados.

Dentre os entrevistados no Município de Campinas que referiram utilizar medicamentos, $30 \%$ obtiveram todos os medicamentos que precisavam por meio do SUS, frequência inferior ao dado nacional encontrado na PNAD 2008, que foi de $45,3 \% 22$ considerando o uso nas duas semanas anteriores à entrevista. Mesmo no contingente da população de Campinas que não tem plano de saúde, o percentual de medicamentos obtidos no SUS atingiu apenas 46,5\%. Esse dado aponta que a maior parte da população de Campinas teve de arcar com os custos dos medicamentos que utilizou, mesmo num contexto em que políticas e programas implementados pelo Estado buscam garantir o acesso universal aos medicamentos incorporados na Relação Nacional de Medicamentos Essenciais (RENAME). Entre as dimensões específicas do acesso, a saber: a disponibilidade física do produto, a acessibilidade geográfica e a aceitabilidade ou satisfação 1; considera-se a disponibilidade física do produto fundamental para garantir o direito constitucional à saúde e ao acesso ao medicamento.

Neste trabalho, foi identificado que dentre os indivíduos que referiram utilizar medicamentos e que os obtiveram por meio do SUS (30\%), a maior frequência foi observada entre aqueles com mais idade, viúvos, não brancos, com menor escolaridade e menor renda familiar per capita, $\mathrm{e}$ entre os que não tinham plano médico de saúde. Dados semelhantes foram verificados em estudo em âmbito nacional, realizado com dados provenientes da PNAD 2008, no qual pouco menos da metade da população que teve os medicamentos prescritos nas últimas duas semanas obteve os mesmos no sistema público de saúde, tendo ocorrido as maiores frequências entre os indivíduos de cor da pele preta, com menor escolaridade e menor renda, e entre aqueles que referiram residir em domicílios cadastrados na Estratégia Saúde da Família 22.

Um aspecto a ser ressaltado é que para uma parcela expressiva de segmentos da população de menor renda, a obtenção gratuita de medicamentos nos serviços públicos de saúde é, frequentemente, a única opção de acesso aos medicamentos 13. Dessa maneira, a gestão e estruturação da assistência farmacêutica na atenção básica necessita ser constante e sistematicamente revista na agenda dos gestores de saúde, de forma a evitar ocorrências de ausência dos medicamentos nos pontos de atenção da rede de saúde.

O percentual elevado do segmento de baixa escolaridade e sem plano de saúde que precisa comprar o medicamento que utiliza é relevante, uma vez que as despesas com medicamentos contribuem de forma decisiva para o gasto total com saúde das unidades familiares do país, e ademais, apresentam tendência de aumento no decorrer do tempo ${ }^{24}$. Entre os setores mais pobres da população, o dispêndio com medicamentos representa a maior parcela dos gastos privados em saúde, e estes gastos se dão de maneira inversamente proporcional à renda, conforme revisão da literatura em 54 países de baixa renda 22,25 .

É importante destacar que no Município de Campinas, na época do estudo, pôde-se verificar que as políticas públicas voltadas à garantia da assistência farmacêutica estão contribuindo para a redução das desigualdades em relação ao acesso a medicamentos, uma vez que foram observadas associações positivas do acesso a medicamentos pelo SUS por indivíduos com menor renda, baixa escolaridade e ausência de cobertura por planos de saúde. Além disso, os resultados demonstram associação positiva com o maior número de doenças crônicas e maior número de medicamentos utilizados, o que pode ser considerado como um indicador positivo para o acesso a medicamentos pelo segmento menos favorecido.

Verificou-se que o percentual da população estudada em Campinas em 2008-2009 que não conseguiu obter os medicamentos prescritos foi bastante reduzido (2,1\%), em comparação com os dados da PMS de 2003 (13\%), o que poderia sugerir que a população adulta de Campinas teria encontrado condições favoráveis no sentido de garantir o acesso ao conjunto dos medica- 
mentos prescritos, no período estudado, mesmo sem obtê-los junto ao SUS.

O presente trabalho possibilitou identificar que, entre os motivos relatados para a não obtenção dos medicamentos prescritos nos últimos 15 dias, está um percentual elevado no tocante à ausência do medicamento no sistema público $(42,5 \%)$. Nesse aspecto, pode-se presumir a ocorrência de distanciamento entre as diretrizes estabelecidas para a assistência farmacêutica na atenção básica e a realidade de municípios brasileiros 26 , uma vez que a disponibilidade de medicamentos na atenção básica está estabelecida nos marcos regulatórios de tal política, sendo esta disponibilidade imprescindível à continuidade dos tratamentos terapêuticos dos pacientes.

A OMS estabelece que a disponibilidade de medicamentos essenciais nos serviços de saúde deve ser superior a $80 \% 27$. Em estudos realizados em cidades do Sul do Brasil, nos quais foi analisada a disponibilidade dos medicamentos nos serviços públicos de saúde, foram encontrados padrões de disponibilidade superiores ao estabelecido pela OMS 24 . Num estudo realizado em cinco estados brasileiros 28 foi registrada a disponibilidade de $74 \%$ de medicamentos essenciais nos serviços de saúde. A análise da disponibilidade de medicamentos essenciais nas unidades públicas de saúde, em municípios mineiros com baixo índice de desenvolvimento humano, foi objeto de outro estudo, no qual foi identificada a disponibilidade de $46,9 \% 29$.

Uma das limitações do presente trabalho é que por ser um estudo transversal não permite a identificação da relação causa e efeito. Além disso, não existe consenso na literatura a respeito do período recordatório ideal para investigar o uso de medicamentos por uma população, pois a seleção do período depende dos aspectos que o investigador pretende pesquisar e da capacidade dos indivíduos em se lembrar. Alguns fatores podem influenciar a maior ou menor retenção das informações, com precisão, tais como o aumento da idade, a presença de doenças e o número de medicamentos utilizados no período 14,30.

Em que pese a implementação de políticas públicas farmacêuticas no país e a ampliação dos gastos federais com a assistência farmacêutica à população, apontadas em diferentes pesquisas 31, como também o estabelecimento da RENAME, sistematicamente atualizada, a qual deve nortear os processos de seleção, prescrição e utilização de medicamentos, o resultado do presente estudo alerta para as dificuldades encontradas na obtenção gratuita de medicamentos, por parte da população de usuários acima de 20 anos, residentes no Município de Campinas no período estudado.

Nesse sentido, este trabalho identificou os segmentos com maior obtenção dos medicamentos do SUS e constatou que mesmo esses subgrupos apresentam porcentuais relativamente baixos de uso de medicamentos do sistema público no município. A partir da realização periódica de inquéritos domiciliares realizados em Campinas, bem como a investigação em outras localidades do país, os resultados permitirão a identificação e comparação de questões relevantes na área, e podem fornecer uma base adequada para a intervenção nessa política pública de saúde.

\section{Colaboradores}

K. S. Costa participou da concepção e delineamento do estudo, análise e interpretação dos dados, redação e revisão crítica relevante do conteúdo intelectual do manuscrito e aprovação da versão a ser publicada. P. M. S. B. Francisco colaborou na da análise e interpretação dos dados e redação do artigo. M. B. A. Barros contribuiu na concepção e delineamento do estudo, análise e interpretação dos dados e aprovação da versão a ser publicada.

\section{Agradecimentos}

Ao CNPq (processo no 409747/2006-8) pelo apoio financeiro ao trabalho de campo do ISACamp. À Secretaria Municipal de Saúde de Campinas e à Secretaria de Vigilância em Saúde do Ministério da Saúde pelo apoio financeiro à realização do inquérito. À FAPESP pela bolsa de pós-doutorado de P. M. S. B. Francisco e ao CNPq pelo financiamento do projeto de pesquisa e pela bolsa de produtividade de M. B. A. Barros. 


\section{Referências}

1. Luiza VL, Bermudez JAZ. Acesso a medicamentos conceitos e polêmicas. In: Bermudez JAZ, Oliveira MA, Escher A, organizadores. Acceso a medicamentos: derecho fundamental, papel del Estado. Rio de Janeiro: Escola Nacional de Saúde Pública; 2004. p. 45-67.

2. Management Sciences for Health. Defining and measuring access to essential drugs, vaccines, and health commodities. In: Report of the WHO-MSH Consultative Meeting. Arlington: Management Sciences for Health; 2000. p. 1-118.

3. Hogerzeil HV, Samson M, Casanovas JV, RahmaniOcora L. Is access to essential medicines as part of the fulfilment of the right to health enforceable through the courts? Lancet 2006; 368:305-11.

4. Bigdeli M, Jacobs B, Tomson G, Laing R, Ghaffar A, Dujardin B, et al. Access to medicines from a health system perspective. Health Policy Plan 2013; 28:692-704.

5. Cameron A, Ewen M, Ross-Degnan D, Ball D, Laing R. Medicine prices, availability and affordability in 36 developing and middle-income countries: a secondary analysis. Lancet 2009; 373:240-9.

6. Ministério da Saúde. Política Nacional de Medicamentos. Brasília: Ministério da Saúde; 2001. (Série C. Projetos, Programas e Relatórios, 25).

7. Ministério da Saúde. Lei no 9.787, de 10 de fevereiro de 1999. Dispõe sobre a vigilância sanitária, estabelece o medicamento genérico, dispõe sobre a utilização de nomes genéricos em produtos farmacêuticos e dá outras providências. Diário Oficial da União 1999; 11 fev.

8. Ministério da Saúde. Resolução no 338, de 06 de maio de 2004. Aprova a Política Nacional de Assistência Farmacêutica e estabelece seus princípios gerais e eixos estratégicos. Diário Oficial da União 2004; 20 mai.

9. Ministério da Saúde. Decreto no 5.090, de 20 de maio de 2004. Regulamenta a Lei no 10.858, de 13 de abril de 2004, e institui o programa "Farmácia Popular do Brasil”, e dá outras providências. Diário Oficial da União 2004; 21 mai.

10. Ministério da Saúde. Portaria no 1.214 de 13 de junho de 2012. Institui o Programa Nacional de Qualificação da Assistência Farmacêutica no âmbito do SUS. Diário Oficial da União 2012; 14 jun.

11. Bertoldi AD, Barros AJD, Hallal PC, Lima RC. Utilização de medicamentos em adultos: prevalência e determinantes individuais. Rev Saúde Pública 2004; 38:228-38.

12. Arrais PSD, Brito LL, Barreto ML, Coelho HLL. Prevalência e fatores determinantes do consumo de medicamentos no Município de Fortaleza, Ceará Brasil. Cad Saúde Pública 2005; 21:1737-46.

13. Paniz VMV, Fassa AG, Facchini LA, Bertoldi AD, Piccini RX, Tomasi E, et al. Acesso a medicamentos de uso contínuo em adultos e idosos nas regiões Sul e Nordeste do Brasil. Cad Saúde Pública 2008; 24:267-80.
14. Costa KS, Barros MBA, Francisco PMSB, César CLG, Goldbaum M, Carandina L. Utilização de medicamentos e fatores associados: um estudo de base populacional no Município de Campinas, São Paulo, Brasil. Cad Saúde Pública 2011; 27:649-58.

15. Rozenfeld S, Valente J. Estudos de utilização de medicamentos: considerações técnicas sobre coleta e análise de dados. Epidemiol Serv Saúde 2004; 13:115-23.

16. Alves MCGP. ISA-Campinas 2008-2009: plano de amostragem. http://www.fcm.unicamp.br/fcm/ sites/default/files/plano_de_amostragem.pdf (acessado em 28/Abr/2014).

17. Barros AJ, Hirakata VN. Alternatives for logistic regression in cross-sectional studies: an empirical comparison of models that directly estimate the prevalence ratio. BMC Med Res Methodol 2003; $3: 21$.

18. Kish L. Survey sampling. New York: John Wiley and Sons; 1965.

19. Carvalho MF, Pascom ARP, Souza-Júnior PRB, Damacena GN, Szwarcwald CL. Utilization of medicines by the Brazilian population, 2003. Cad Saúde Pública 2005; 21 Suppl 1:S100-8.

20. Boing AC, Bertoldi AD, Peres KG. Desigualdades socioeconômicas nos gastos e comprometimento da renda com medicamentos no Sul do Brasil. Rev Saúde Pública 2011; 45:897-905.

21. Hovstadius B, Astrand B, Petersson G. Dispensed drugs and multiple medications in the Swedish opulation: an individual-based register study. BMC Clin Pharmacol 2009; 9:11

22. Boing AC, Bertoldi AP, Boing AF, Bastos JL, Peres KG. Acesso a medicamentos no setor público: análise de usuários do Sistema Único de Saúde no Brasil. Cad. Saúde Pública 2013; 29:691-701.

23. Ribeiro AQ, Rozenfeld S, Klein CH, César CC, Acurcio FA. Inquérito sobre o uso de medicamentos por idosos aposentados, Belo Horizonte, MG. Rev Saúde Pública 2008; 42:724-32.

24. Hefler AP, Tavares NU, Kanavos P, Bertoldi AD. Capacidade aquisitiva e disponibilidade de medicamentos para doenças crônicas no setor público. Rev Panam Salud Pública 2012; 31:225-32.

25. Vialle-Valentin CE, Ross-Degnan D, Ntaganira J, Wagner AK. Medicines coverage an communitybased health insurance in low-income countries. Health Res Policy Syst 2008; 6:11.

26. Oliveira LCF, Assis MMA, Barboni AR. Assistência farmacêutica no Sistema Único de Saúde: da Política Nacional de Medicamentos à atenção básica à saúde. Ciênc Saúde Coletiva 2010; 15 Suppl 3:3561-7.

27. World Health Organization. Continuity and change. Implementing the third WHO Medicines Strategy 2008-2013. http://apps.who.int/medi cinedocs/documents/s16880e/s16880e.pdf (acessado em 28/Abr/2014). 
28. Organização Pan-Americana da Saúde. Avaliação da assistência farmacêutica no Brasil: estrutura, processo e resultados. Brasília: Organização PanAmericana da Saúde; 2005.

29. Guerra Jr. AA, Acurcio FA, Gomes CAP, Miralles M, Girardi SN, Werneck GAF. Disponibilidade de medicamentos essenciais em duas regiões de Minas Gerais, Brasil. Rev Panam Salud Pública 2004; 15:168-75.

\begin{abstract}
The use of medicines is influenced by different factors. This study aimed to analyze the use of medicines and identify the sources for obtaining them and associated factors, using a population-based cross-sectional design with two-stage cluster sampling in Campinas, São Paulo State, Brazil, in 2008. Prevalence of use of at least one drug in the 3 previous days was $57.2 \%$ and prevalence of obtaining the drug from the Brazilian Unified National Health System (SUS) was 30\%. $47.8 \%$ of individuals with no health insurance had to pay out-of-pocket for their medication, and $10.9 \%$ of individuals with insurance nevertheless obtained their medication from the SUS. Use of medicines obtained from the SUS was associated with older age, widowhood, black or brown skin color, lower schooling and income, and lack of insurance. Only $2.1 \%$ reported not being able to obtain the prescribed medication, and the main reasons were lack of availability in the public system and lack of money for out-of-pocket purchases. The study identified the subgroups that obtained most of their medicines in the SUS and showed that these were the subgroups with the lowest use of medicines in the system.
\end{abstract}

Pharmaceutical Services; Drug Utilization; Health Surveys
30. Bertoldi AD, Barros AJD, Wagner A, Rooss-Degnan D, Hallal PC. A descriptive review of the methodolo-gies used in household surveys on medicine utilization. BMC Health Serv Res 2008; 8:222.

31. Aurea AP, Magalhães LCG, Garcia LP, Santos CF, Almeida RF. Programas de assistência farmacêutica do governo federal: estrutura atual, evolução dos gastos com medicamentos e primeiras evidências de sua eficiência, 2005-2008. Brasília: Instituto de Pesquisa Econômica Aplicada; 2011. (Textos para Discussão, 1658).

\section{Resumen}

El uso de medicamentos está influenciado por diversos factores en la sociedad. El objetivo fue analizar el uso de fármacos e identificar las fuentes de obtención $y$ factores asociados. Se trata de un estudio transversal de base poblacional, con muestreo por conglomerados, en dos etapas, en Campinas, São Paulo, Brasil, 2008. La prevalencia del uso de al menos un medicamento, en los 3 días previos fue de 57,2\%; la prevalencia de obtención en el Sistema Único de Salud (SUS) fue de $30 \%$. Cerca de 47,8\% de los individuos sin seguro tuvieron que pagar para adquirir los medicamentos $y$ un 10,9\% de los asegurados los obtuvieron en el SUS. Utilizaron con mayor frecuencia los fármacos del SUS los individuos de mayor edad, viudos, negros/mulatos con menor escolaridad y renta y no asegurados. Apenas un 2,1\% de la población entrevistada alegó no haber logrado obtener un medicamento prescrito y los principales motivos fueron no estar en el sistema público y no tener recursos. El estudio identifica los segmentos con mayor obtención a los fármacos del SUS y revela que estos subgrupos presentan bajos porcentajes de uso de medicamentos en el sistema.

Servicios Farmacéuticos; Utilización de Medicamentos; Encuestas Epidemiológicas
Recebido em 02/Mai/2014

Versão final reapresentada em 01/Jun/2015 Aprovado em 08/Jun/2015 